\title{
Shape Memory Properties of Ni-Ti Based Melt-Spun Ribbons
}

\author{
R. SANTAMARTA, E. CESARI, J. PONS, and T. GORYCZKA
}

\begin{abstract}
Shape-memory properties of equiatomic NiTi, $\mathrm{Ni}_{45} \mathrm{Ti}_{50} \mathrm{Cu}_{5}$, and $\mathrm{Ni}_{25} \mathrm{Ti}_{50} \mathrm{Cu}_{25}$ ribbons made by melt spinning have been studied by temperature inducing the martensitic transformation under constant tensile loads. Recoverable strains above 4 pct can be obtained under $\sim 100 \mathrm{MPa}$ loads for the NiTi and $\mathrm{Ni}_{45} \mathrm{Ti}_{50} \mathrm{Cu}_{5}$ ribbons, transforming to $\mathrm{B} 19^{\prime}$ martensite. The $\mathrm{B} 19$ martensite is formed in the $\mathrm{Ni}_{25} \mathrm{Ti}_{50} \mathrm{Cu}_{25}$ ribbon after crystallization, and according to the lowering in transformation strain as $\mathrm{Cu}$ content increases, the recoverable strain is close to $2.5 \mathrm{pct}$ for $\sim 150 \mathrm{MPa}$ load. The transformation temperatures exhibit a linear dependence on the applied stress, which can be quantitatively described by means of a Clausius-Clapeyron type equation. The NiTi and $\mathrm{Ni}_{45} \mathrm{Ti}_{50} \mathrm{Cu}_{5}$ ribbons exhibited some degree of two-way shape-memory effect (TWSME) after thermomechanical cycling. Texture analyses performed on the different ribbons allow us to better understand the transformation strains obtained in each ribbon. The amounts of shapememory effect (SME) and nonrecoverable strain shown by the studied ribbons are of the same order as those already observed in bulk materials, which makes melt spinning an ideal substitute to complicated manufacturing processes if really thin samples are needed. However, applicable stresses in melt-spun ribbons are limited by a relatively "premature" brittle fracture caused by irregularities in ribbon thickness.
\end{abstract}

\section{INTRODUCTION}

NI-TI shape-memory alloys (SMAs), and especially nearequitomic Ni-Ti, show very good mechanical and functional properties (large recovery strains and stresses, high corrosion resistance, good fatigue properties, and biocompatibility), being the most widely used SMA system for applications with transformation temperatures close to room temperature. However, some features of these alloys are not suitable for all the present or potential new applications. This has led to the study of additions of a third or more elements to the binary $\mathrm{Ni}-\mathrm{Ti}$ or to the use of nonconventional production techniques, in order to modify the martensitic transformation temperatures, hysteresis, and the thermal stability of the alloys, to improve some mechanical properties, etc. ${ }^{[1-4]}$

Substituting $\mathrm{Cu}$ for $\mathrm{Ni}$ in the binary Ni-Ti lowers the transformation hysteresis, which can be beneficial for some applications, and decreases the effect of cycling processes in the transformation ${ }^{[5,6]}$ without modifying significantly the transformation temperatures. The addition of copper also reduces the sensitivity of the transformation temperatures to compositional changes and prevents $\mathrm{Ti}_{3} \mathrm{Ni}_{4}$ precipitation, ${ }^{[5]}$ but it may produce a change in the transformation sequence. For $\mathrm{Cu}$ amounts not much higher than 5 at. pct, the B2 parent phase transforms to monoclinic B19' martensite (such as in the near equiatomic $\mathrm{Ni}-\mathrm{Ti}$ ). In the range from 5 to 15 at. pct, the transformation sequence is often split into B2 $\rightarrow$ orthorhombic (B19) $\rightarrow$ B19'; the increase in $\mathrm{Cu}$ content shifts the B19 $\rightarrow$ B19' transformation to lower temperatures. For contents of copper higher than 15 at. pct, the transformation B19 $\rightarrow$ B19' takes place at so low a temperature that only the B2 $\rightarrow$ B19 is usually observed. ${ }^{[5]}$

R. SANTAMARTA, Lecturer, E. CESARI, Professor, and J. PONS, Assistant Professor, are with the Department of Physics, University of Illes Balears, E-07122 Palma de Mallorca (Illes Balears), Spain. Contact e-mail: ruben.santamarta@uib.es T. GORYCZKA, Lecturer, is with the Institute of Physics and Chemistry of Metals, University of Silesia, 40007 Katowice, Poland.

Manuscript submitted December 30, 2002.
Recently, a growing interest in obtaining high-transformation temperatures in the Ni-Ti system has promoted the addition of third elements such as $\mathrm{Hf}$ or $\mathrm{Zr}$, much cheaper than those previously used for that purpose ( $\mathrm{Au}, \mathrm{Pd}$, and $\mathrm{Pt})$. Those elements have shown an effective increasing of the transformation temperatures over $575 \mathrm{~K}$ for amounts higher than 20 at. pct, ${ }^{[7,8]}$ but they have also shown a poor stability upon thermal treatments even at temperatures slightly higher than $A_{f}$ or in martensite. ${ }^{[9,10]}$

Another way to enlarge the range of applications of SMAs, apart from the addition of extra elements to the Ni-Ti system, is the use of nonconventional production techniques for casting, such as rapid solidification by melt spinning. Although this technique is not new, its application to SMAs is relatively recent. Probably the best advantage of this technique is that almost ready-to-use ribbons are directly produced, avoiding the typical thermomechanical shaping procedures needed after conventional casting, thus leading to near-net-shape SMAs for certain applications. On the other hand, melt spinning introduces microstructural differences in comparison with the conventional cast material; the best known example is the reduction of the grain size (in some cases, the as-spun ribbons may be almost fully amorphous) promoting a decrease in the transformation temperatures and a possible improvement of mechanical and functional properties (i.e., superelasticity and damping capacity). ${ }^{[11,12]}$ However, not many references about the mechanical behavior or the shape-memory effect (SME) shown by melt-spun ribbons can be found. The present work will study more deeply the shape memory behaviour of equiatomic $\mathrm{NiTi}$, and $\mathrm{Ni}-\mathrm{Ti}-\mathrm{Cu}$ (with $\mathrm{Cu}$ content of 5 and 25 at. pct) melt spun ribbons and will be compared to results already reported for bulk material. The shape-memory properties have been studied through the temperature-induced transformation under constant load (thermomechanical cycling). X-ray experiments have been performed in order to make measurements of the texture and preferred orientations of the crystals in these ribbons. Eventual changes in the microstructure have been checked by transmission electron microscopy. 


\section{EXPERIMENTAL PROCEDURE}

The studied melt-spun ribbons were prepared under helium atmosphere by planar flow casting onto a rotating $\mathrm{Cu}$ or steel wheel from conventionally produced ingots of the corresponding alloys. Nominal compositions and some melt-spinning parameters, as well as the mean thickness of the ribbons, are shown in Table I. All the ribbons have good macroscopic quality, tens of centimeters in length, their width ranging from $9 \mathrm{~mm}$ for the equiatomic $\mathrm{NiTi}$ and $\mathrm{Ni}_{45} \mathrm{Ti}_{50} \mathrm{Cu}_{5}$ to $21 \mathrm{~mm}$ for the $\mathrm{Ni}_{25} \mathrm{Ti}_{50} \mathrm{Cu}_{25}$. The $\mathrm{NiTi}$ and $\mathrm{Ni}_{45} \mathrm{Ti}_{50} \mathrm{Cu}_{5}$ ribbons have been checked in as-spun condition, whereas the $\mathrm{Cu}-25$ ribbon, which is initially almost completely amorphous, has been crystallized by heating the samples at $880 \mathrm{~K}$ for 3.5 minutes previously to the thermomechanical tests. Differential scanning calorimetry PerkinElmer Inc., Norwalk, Connecticut (CT), USA (PerkinElmer DSC-7) has been used to determine the transformation temperatures and the enthalpy and entropy changes.

Several series of thermomechanical cycles at different levels of constant tensile load (up to $150 \mathrm{MPa}$ ) have been carried out in a specially designed device. The specimen, whose distance between grips is around $2-\mathrm{cm}$ long, has been heated/cooled in a liquid bath repeatedly, while its length variation has been measured with a linear variable differential transducer (LVDT) sensor, in order to characterize the SME. When mounting naked ribbons in the device, a premature fracture occurred with relatively high frequency in the regions where the grips hold the ribbons. The use of aluminum foil covers in these regions has improved the fracture stresses during the experiments. After some thermomechanical cycles performed at different stresses, the two-way shape-memory Effect (TWSME) has been investigated, as well.

Tensile tests have been carried out in some $\mathrm{Cu}-25$ samples using a Zwick GmbH \& Co. KG, Ulm, Germany, Zwick Z100/ TL3A machine with a digital extensometer and a controlled temperature chamber, determining the Young modulus in martensite and austenite and obtaining superelastic cycles for this alloy.

$\mathrm{X}$-ray measurements of preferred orientations have been done with a PHILIPS* PW1130 diffractometer operating

*PHILIPS is a trademark of Philips Electronic Instruments Corp., Mahwah, NJ.

with $\mathrm{Cu} K_{\alpha}$ radiation and equipped with a texture goniometer. Incomplete pole figures were recorded in reflection mode with a maximum tilting angle of $80 \mathrm{deg}$. Intensity was corrected using the Schulz formula.

Microstructure observations for the as-spun and cycled ribbons have been performed in a Hitachi H-600 transmission electron microscope (TEM) operating at $100 \mathrm{kV}$ and in a JEOL*-2011 high-resolution TEM at $200 \mathrm{kV}$.

*JEOL is a trademark of Japan Electron Optics Ltd., Tokyo.

Table I. Composition, Melt-Spinning Parameters, and Thickness of the Studied Ribbons

\begin{tabular}{lccc}
\hline Alloy & $\mathrm{NiTi}$ & $\mathrm{Cu}-5$ & $\mathrm{Cu}-25$ \\
\hline Composition (at. pct) & $\mathrm{Ni}_{50} \mathrm{Ti}_{50}$ & $\mathrm{Ni}_{45} \mathrm{Ti}_{50} \mathrm{Cu}_{5}$ & $\mathrm{Ni}_{25} \mathrm{Ti}_{50} \mathrm{Cu}_{25}$ \\
Melting temperature $(\mathrm{K})$ & 1740 & 1700 & 1625 \\
Wheel speed (m/s) & 19 & 19 & 23 \\
He ejection pressure (hPa) & 220 & 200 & 200 \\
Thickness $(\mu \mathrm{m})$ & $40 \pm 3$ & $41 \pm 5$ & $35 \pm 3$ \\
\hline
\end{tabular}

\section{RESULTS AND DISCUSSION}

Before describing the results obtained for the melt-spun alloys, it must be said that several thermomechanical series (increasing external stress) have been performed on each ribbon. Total strain and transformation temperatures calculated with the tangent method have been worked out for each stress, showing good reproducibility for both magnitudes. This reproducibility is lower when samples are dismounted and mounted again. Although the cycles performed to the same or different specimens of the same alloy under the same conditions are practically indistinguishable, it has to be noted that the total strains recorded on cooling and heating branches may differ slightly. This is due to the deviations from linearity in the LVDT sensor below $270 \mathrm{~K}$ and to the different temperature gradients between the sample and the system LVDT-sample holder when increasing and decreasing the temperature. As a consequence of this, curvatures of the strain-temperature plots in the region below $\sim 260 \mathrm{~K}$ and nonsymmetrical thermal dilatation effects between cooling and heating curves take place; none of these effects has been corrected in the thermomechanical cycles shown subsequently (Sections A-C). Apart from this, it should be noted that in some cases it is possible to observe a residual strain when a complete cooling-heating cycle is run, even under low stresses. The appearance of this effect is connected with the existence of temperature gradients in the sensor system promoting delays when getting to the initial state, especially after reaching low temperatures. Part of this effect is also related to a real plastic deformation of the sample, mainly at high stresses.

\section{A. NiTi Ribbons}

The strain-temperature cycles under loads below $80 \mathrm{MPa}$ show two-step transformation on cooling (the first one appearing as a small "kink" in the strain-temperature curve, at low strains) and a single one during heating (Figure 1), as has also been observed in the characterization by DSC. ${ }^{[13]}$ However, cooling curves at increasing loads show the approaching of both steps until they are indistinguishable (Figure 1). Figure 2 shows that this effect is due to the dif-

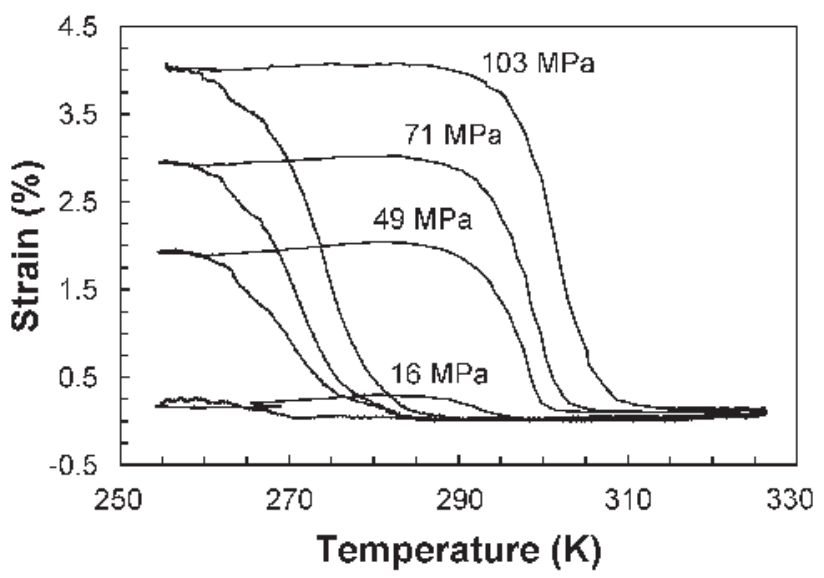

Fig. 1-Selection of thermal cycles performed under growing stresses in the equiatomic ribbon. The transformation to $\mathrm{R}$ phase is visible at the beginning of the cooling branch. 
ferent dependence of the transformation temperatures $\left(M_{s}\right.$ for the $\mathrm{R} \rightarrow \mathrm{B} 19^{\prime}$, and $R_{s}$ for the $\mathrm{B} 2 \rightarrow \mathrm{R}$ ) on the applied stress, i.e., the shift is larger for the $\mathrm{R} \rightarrow \mathrm{B} 19^{\prime}$ than for the $\mathrm{B} 2 \rightarrow \mathrm{R}$ transformation. This means that over a critical external stress, the original transformation sequence $(\mathrm{B} 2 \rightarrow \mathrm{R} \rightarrow$ B19') becomes a single-step sequence $\left(\mathrm{B} 2 \rightarrow \mathrm{B} 19^{\prime}\right)$. The slope $d T / d \sigma$, which characterizes the dependence of the transformation temperatures on the external stress, can be either calculated by a least-squares fit (as for the values shown in Figure 2) or estimated by means of a Clausius-Clapeyron type equation using data from calorimetric measurements and strains obtained in thermomechanical cycling. This equation is equivalent to the well-known equation for $d \sigma / d T$ (from now on, we will use the form $d \sigma / d T$, more widely used than $d T / d \sigma$ ) giving the temperature dependence of the critical stress in stress-induced transformations at different temperatures. ${ }^{[14]}$ For a transformation from a parent phase "p" to a martensitic phase "m," the Clausius-Clapeyron type equation can be written as

$$
\frac{d \sigma^{p \rightarrow m}}{d T}=-\frac{\rho \Delta S}{\varepsilon}
$$

where $\Delta S$ is the entropy change of the transformation, $\varepsilon$ the maximum recoverable strain during the thermomechanical cycles, and $\rho$ the density of the alloy. It is also possible to use another form for the previous equation:

$$
\frac{d \sigma^{p \rightarrow m}}{d T}=-\frac{\rho \Delta H}{\varepsilon T_{0}}
$$

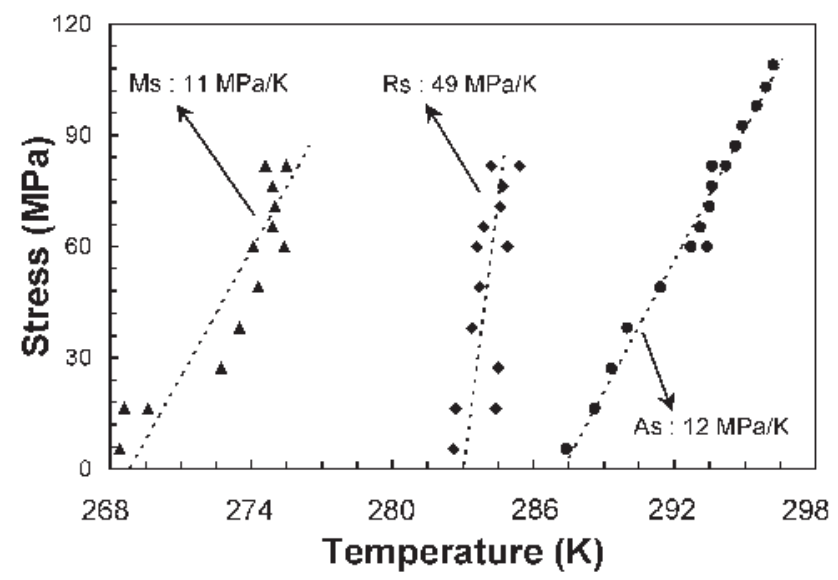

Fig. 2-Transformation temperatures $v s$ external applied stress for the NiTi. The experimental value of the slope $d \sigma / d T$ in the Clausius-Clapeyon equation type is also indicated. where $\Delta H$ is the enthalpy change and $T_{0}$ is the temperature of equilibrium between the two phases, which is usually taken as $\left(M_{s}+A_{f}\right) / 2$. However, the peculiar transformation sequence of the binary ribbon makes this formula not applicable, as there is no a separated reverse transformation for the $\mathrm{B} 19^{\prime}$ and $\mathrm{R}$ phases. In turn, $T_{0}$ has been taken simply as the transformation temperatures $R_{s}, M_{s}$, and $A_{s}$, for the

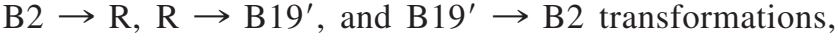
respectively. The results obtained from the preceding equations are quite similar to each other and to those obtained by fitting the experimental data, as shown in Table II. Any set of $d \sigma / d T$ values in Table II shows a considerably higher slope for the B2 $\rightarrow \mathrm{R}$ than for the $\mathrm{R} \rightarrow \mathrm{B} 19^{\prime}$. Therefore, for the same stress, the transformation temperatures will be more shifted in the $\mathrm{R} \rightarrow \mathrm{B} 19^{\prime}$ transformation and will overpass the $\mathrm{B} 2 \rightarrow \mathrm{R}$ when increasing the stress, as has been observed experimentally (Figure 1).

From a physical point of view, the application of an external stress promotes a relatively smaller movement of the Gibbs free energy for the R phase compared to the B19' phase, both in relation to B2, and so the effect on the transformation temperatures. Upon increasing stress, the Gibbs energies will be correspondingly shifted until $M_{s}$ becomes higher than $R_{s}$, leading to the single-step transformation $\mathrm{B} 2 \rightarrow \mathrm{B} 19^{\prime}$.

The evolution of the transformation strain on cooling as a function of the applied stress for a NiTi specimen cycled from the as-spun state, in consecutive series at increasing loads up to $110 \mathrm{MPa}$, is shown in Figure 3. The split of the direct transformation into $\mathrm{B} 2 \rightarrow \mathrm{R}$ and $\mathrm{R} \rightarrow \mathrm{B} 19^{\prime}$ turns out to be below the resolution of the device when the stress is above $80 \mathrm{MPa}$, so only the total strain is represented in Figure 3 for higher stresses. When both stages of the transformation sequence are distinguishable, it is clear that the largest contribution to the total strain corresponds to the R $\rightarrow$ B19' transformation. The reason is that the distortion involved in the $\mathrm{B} 2 \rightarrow \mathrm{R}$ is much lower than in the $\mathrm{R} \rightarrow \mathrm{B} 19^{\prime}$; the lattice deformation associated to the $\mathrm{R}$ phase cannot be higher than 0.94 pct (although experimental values are usually below $\left.0.8 \mathrm{pct}^{[15,16]}\right)$, whereas even one order of magnitude larger deformations can be obtained for the B19' phase ${ }^{[5]}$ However, in a polycrystalline material, the interaction between grains, precipitation effects, or preferred orientations can play an important role in this sense and modify considerably these values. Sittner and Novák ${ }^{[17]}$ have calculated the deformation for a B2 $\rightarrow$ B19' transformation in a polycrystalline NiTi by means of simulations taking into account only an "average" of different orientations, obtaining values between 8.6 and 5.7 pct. Moreover, a high dispersion of experimental results exists for near-equiatomic polycrystalline NiTi

Table II. Values for the $d \sigma / d T$ Slopes Worked Out from Equations [1] and [2] and Obtained

\begin{tabular}{|c|c|c|c|c|c|c|c|}
\hline Transformation & $\begin{array}{c}\Delta S \\
\left(\mathrm{~J} \cdot \mathrm{kg}^{-1} \cdot \mathrm{K}^{-1}\right)\end{array}$ & $\begin{array}{c}\Delta H \\
\left(\mathrm{~J} \cdot \mathrm{kg}^{-1}\right)\end{array}$ & $T_{0}(\mathrm{~K})$ & $\varepsilon$ & $\begin{array}{c}(d \sigma / d T)_{1} \\
\left(\mathrm{MPa} \cdot \mathrm{K}^{-1}\right)\end{array}$ & $\begin{array}{c}(d \sigma / d T)_{2} \\
\left(\mathrm{MPa} \cdot \mathrm{K}^{-1}\right)\end{array}$ & $\begin{array}{c}(d \sigma / d T)_{\exp } \\
\left(\mathrm{MPa} \cdot \mathrm{K}^{-1}\right)\end{array}$ \\
\hline $\mathrm{B} 2 \rightarrow \mathrm{R}$ & $\approx-20$ & $\approx-4500$ & $\approx 291$ & 0.002 & 65 & 50 & 49 \\
\hline $\mathrm{R} \rightarrow \mathrm{B} 19^{\prime}$ & $\approx-50$ & $\approx-16,100$ & $\approx 273$ & 0.038 & 8.6 & 10.1 & 11 \\
\hline $\mathrm{B} 19^{\prime} \rightarrow \mathrm{B} 2$ & 70 & 20,600 & $\approx 309$ & 0.040 & 11.4 & 10.8 & 12 \\
\hline
\end{tabular}
by Fitting the Experimental Data*

*The thermodynamical magnitudes used for calculations are also included. A symbol $\approx$ is shown when these are roughly estimated due to the slight overlapping of the two calorimetric peaks. Density has been set as $\rho=6500 \mathrm{~kg} \cdot \mathrm{m}^{-3}$. 


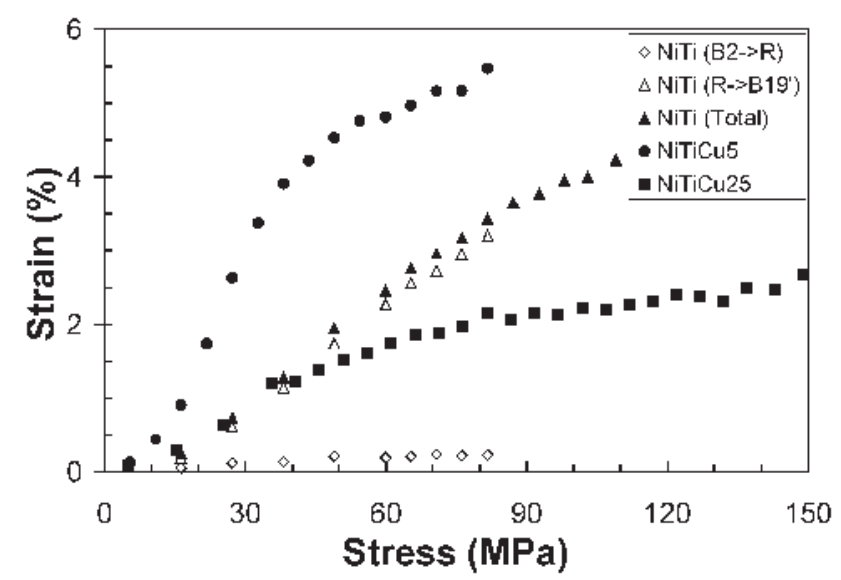

Fig. 3-Maximum strain achieved for all the melt-spun ribbons (including nonrecoverable strain) during the first time the corresponding stress has been applied.

alloys, ${ }^{[18]}$ most of them being not very different from the maximum recoverable strain attained for the NiTi melt-spun alloy in this work $(\approx 4 \mathrm{pct})$. This is a good value, especially if we take into account the lower grain size of the melt-spun ribbons and the relatively low maximum stress applicable for the melt-spun ribbons before the brittle fracture occurs, which hinders the reaching of the saturation of the SME. For the equiatomic ribbon, the fracture stress lies between 90 and $110 \mathrm{MPa}$, which are low values compared to the bulk material. This brittleness has to be related to stress concentrations, which take place in inhomogeneities of the ribbons, such as voids or thickness irregularities (the thinner areas of the material, where the stresses are concentrated, promote macroscopic fracture). Propagation and growth of microcracks during cycling at high stresses could be a determinant factor as well. In this sense, increasing the thickness of the melt-spun ribbons may considerably improve the quality of their mechanical behavior, lowering the probability for the existence of very thin regions, which is determinant for the mentioned fracture at low stresses.

Another difference between the B2 $\rightarrow \mathrm{R}$ and $\mathrm{R} \rightarrow \mathrm{B} 19^{\prime}$ transformations observed in Figure 3 is that the former one reaches a saturation strain value close to 0.3 pct for stress levels about $70 \mathrm{MPa}$, but not the latter one, resulting in no saturation of the total SME up to $110 \mathrm{MPa}(\varepsilon \sim 4 \mathrm{pct})$, as was previously mentioned. In addition, some residual deformation $\sim 0.2$ pct has been detected at these stress levels, which are values comparable to the ones obtained for bulk material.

Thermomechanical cycling is not only useful to measure some shape-memory properties, but also an easy way to induce the TWSME. In order to perform these experiments, several cycles at the lowest stress levels (those needed to keep the specimen properly aligned) have been performed in samples previously cycled. The example in Figure 4 shows a strain close to 1 pct for a sample with 50 cycles carried out in three series under increasing loads up to $100 \mathrm{MPa}$, whereas only a third part of this value was obtained in samples without previous cycling. The TWSME obtained for the NiTi melt-spun ribbon is comparable to that obtained in conventionally cast alloys. In these experiments, some differences between the first and second parts of the trans-

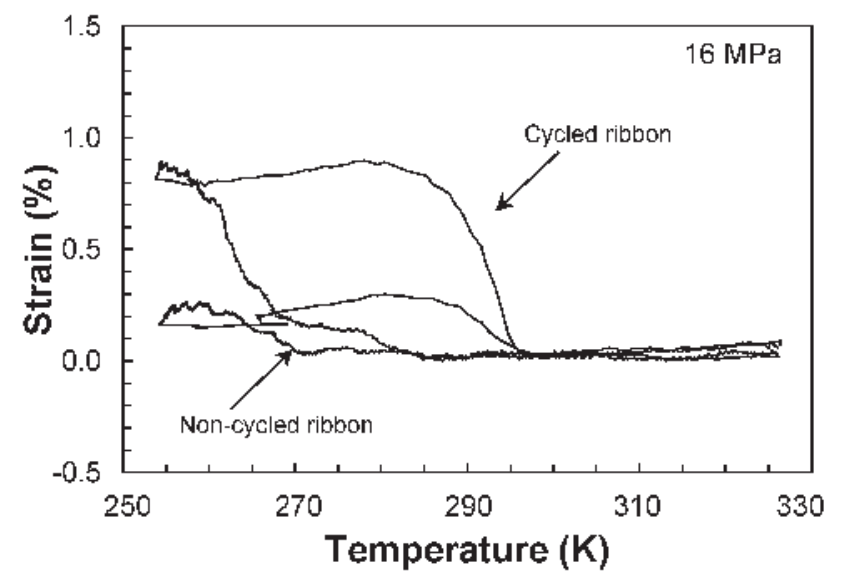

Fig. 4-Thermomechanical cycles performed in a NiTi sample previously cycled (50 times with increasing loads up to $100 \mathrm{MPa}$ ) and a noncycled specimen, which clearly show the presence of TWSME.

formation may also be observed when comparing Figure 4 and Figure 1 or 2 . The first part of the transformation (B2 $\rightarrow$ $\mathrm{R})$ of the cycled specimen is close to its saturation strain, whereas the second part, $\mathrm{R} \rightarrow \mathrm{B} 19^{\prime}$, is still far from its maximum value. This points to a higher easiness to induce the TWSME in the R phase than in the B19' martensite. During the cycling procedure, the configuration of the existing dislocations may change (or some of them may be created) in such a way that the selection of the variants favored by the applied stress is more strongly promoted in the $\mathrm{R}$ phase than in the B19', reaching the saturation value faster.

To clarify the presence of texture in the equiatomic ribbon, pole figures of $\{110\}_{\mathrm{B} 2},\{100\}_{\mathrm{B} 2}$, and $\{211\}_{\mathrm{B} 2}$ were registered at room temperature in the range 0 to $80 \mathrm{deg}$ (Figure 5). Indexes refer to the parent phase (B2), which is the one observed at room temperature. In this ribbon, two components of the texture are observed: the $\{100\}$ axial texture and the $(001)<100>$ sheet texture. Generally, texture is tilted in the horizontal and vertical directions according to the wheel rotation direction (RD). The maximum normalized intensity is 24.7 and relates to the axial texture, whereas the maximum of the sheet texture component equals 2.9. Despite the fact that the axial texture is much higher, the presence of a shifted texture could hinder the strain in such a way that it makes it difficult to reach higher values in the direction of the applied stress (in the equiatomic ribbon, the maximum recoverable strain obtained in the experiments is in the middle range of the values obtained for Ni-Ti bulk alloys). A "welloriented" axial texture would promote reaching the saturation strain in the SME (and to approach the maximum value predicted by the theory) due to the promotion of a higher amount of proper variants leading to higher macroscopic deformation.

The microstructure in the initial state (without any thermal treatment) observed by TEM consists mainly of elongated grains (the longest grains observed being $\sim 15-\mu \mathrm{m}$ long and $\sim 1-\mu \mathrm{m}$ wide), although it is possible to find more spheroidal ones (with a radius of a few micrometers) as well. Grain boundaries are wavy and irregular and a high density of severely entangled dislocations may be found inside the grains. Additionally, very small precipitates $(\sim 10-\mathrm{nm}$ size $)$ immersed in the dislocation tangles can also be observed. The small size of the precipitates makes difficult their iden- 

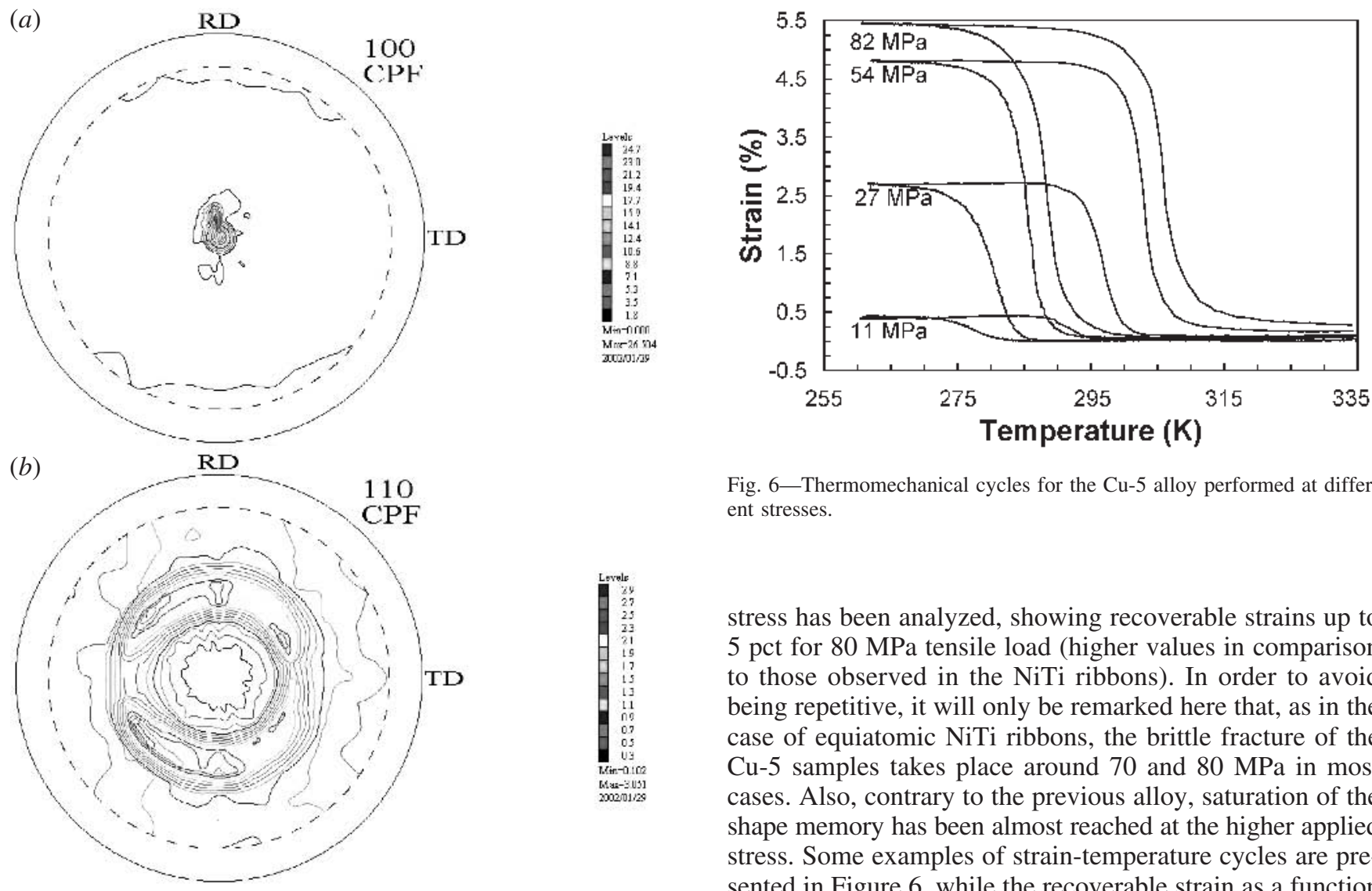

Fig. 6-Thermomechanical cycles for the $\mathrm{Cu}-5$ alloy performed at different stresses.

stress has been analyzed, showing recoverable strains up to 5 pct for $80 \mathrm{MPa}$ tensile load (higher values in comparison to those observed in the NiTi ribbons). In order to avoid being repetitive, it will only be remarked here that, as in the case of equiatomic NiTi ribbons, the brittle fracture of the $\mathrm{Cu}-5$ samples takes place around 70 and $80 \mathrm{MPa}$ in most cases. Also, contrary to the previous alloy, saturation of the shape memory has been almost reached at the higher applied stress. Some examples of strain-temperature cycles are presented in Figure 6, while the recoverable strain as a function of the applied stress plot is shown in Figure 3. By comparing the cycles in Figure 6 and those in Figure 1, it is worth noting the effect of $\mathrm{Cu}$ content in reducing the transformation hysteresis, a well-known effect. ${ }^{[5]}$ Residual deformations below 0.2 pet have been observed in $\mathrm{Cu}-5$ ribbons, lower than in commercial wires $(\sim 0.6$ pct at $70 \mathrm{MPa}$ or 1.8 pct under $110 \mathrm{MPa}^{[20]}$ ). Thus, this set of results indicates that the functional performance is somewhat higher in this ribbon than in the NiTi one.

A linear dependence of the transformation temperatures as a function of the external stress has also been obtained in this ribbon; its slope $d \sigma / d T$ has been estimated from the already described methods. In this case, the equilibrium temperature has been taken in the usual way $T_{0}=\left(M_{s}+A_{f}\right) / 2$. The theoretical and experimental results are moderately different, i.e., 9.9, 9.2, and $6 \mathrm{MPa} / \mathrm{K}$ using Eqs. [1], [2], or the experimental fitting, respectively, for the forward transformation.

As is shown in Figure 7, the TWSME induced by thermomechanical cycling has been measured after $\sim 75$ cycles up to $80 \mathrm{MPa}$, giving a $\sim 0.7$ pct recoverable deformation under $5 \mathrm{MPa}$ (very low stress, just to keep the ribbon extended) and close to 1.8 pct under $16 \mathrm{MPa}$; the analogous values for noncycled ribbons are less than 0.2 and $1 \mathrm{pct}$ respectively. Although these values are slightly smaller than for the equiatomic ribbon, the value obtained under $16 \mathrm{MPa}$ is comparable to those reported for commercial wires with 10 at. pct $\mathrm{Cu}^{[20]}$

In this composition, only axial texture has been found. According to the pole figures registered for $(110)_{\mathrm{B} 2},(100)_{\mathrm{B} 2}$, and $(211)_{\mathrm{B} 2}$ peaks (Figure 8), the normalized intensity is smaller than in the NiTi (the maximum being around 10.5); additionally, the center of $\{100\}_{\mathrm{B} 2}$ axial texture is tilted about 


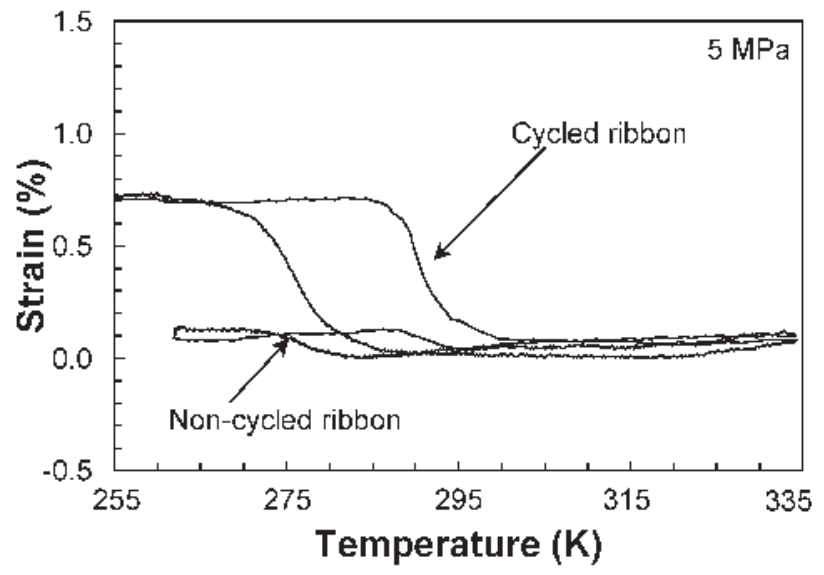

Fig. 7-TWSME for the $\mathrm{Cu}-5$ ribbon tested under $5 \mathrm{MPa}$ stress. The cycled sample has undergone 75 previous cycles with stresses lower than $85 \mathrm{MPa}$.

$10 \mathrm{deg}$ in the wheel RD. Although the texture is lower in this ribbon, the strain obtained in the thermomechanical cycling under the same stress is higher than in the NiTi. This means that either the sheet texture present in the previous alloy or the tilting of the axial texture in the wheel rotation direction in the $\mathrm{Cu}-5$ alloy hinders or promotes, respectively, variants with maximum strain along the stress direction, giving a higher macroscopic strain during thermomechanical cycling. At this point, the angle of the monoclinic cell (B19') should be taken into account: having a $\{100\}_{\mathrm{B} 2}$ axial texture means that there are no variants with the maximum deformation completely aligned in the direction of the applied stress; tilting the texture a few degrees could increase the possibilities to find a better orientation of the variants. Therefore, the higher strain reached in $\mathrm{Cu}-5$ under similar external stresses in comparison to the equiatomic one is most likely due to this tilt in the texture, which was absent in the previous alloy.

The microstructure of the ribbons containing 5 at. pct of copper do not show grains as elongated as in the NiTi case, their shapes being more rounded (with diameters around 2 to $3 \mu \mathrm{m})$ and exhibiting wavy and irregular boundaries. The as-spun ribbons contain a dispersion of spheroidal precipitates of higher density than that observed in the as-received $\mathrm{NiTi}$, the precipitates also being larger in size (about 20 to $30 \mathrm{~nm}$ for the larger ones). Many dislocations have been observed, usually joining precipitates, although in a lower amount than in the equiatomic alloy and not grouped in entangled networks.

\section{Cu-25 Ribbons}

The as-spun microstructure of this alloy is quite different from those studied above: X-ray diffraction (XRD) and TEM experiments have shown that it is mainly amorphous. According to a quantitative analysis carried out by means of Rietveld refinement, the presence of the amorphous phase rises to 99 pct, whereas the rest of the sample is in the B2 phase (reliability factors of $R_{p}=3.04 \mathrm{pct}$ and $R_{w p}=3.81 \mathrm{pct}$ ). This low amount of B2 is in agreement with the fact that no peaks attributed to the martensitic transformation are detected in the differential scanning calorimetry within the range 235 to $870 \mathrm{~K}$. Moreover, the intensity distribution of the XRD peaks shows the crystallized grains oriented according to the
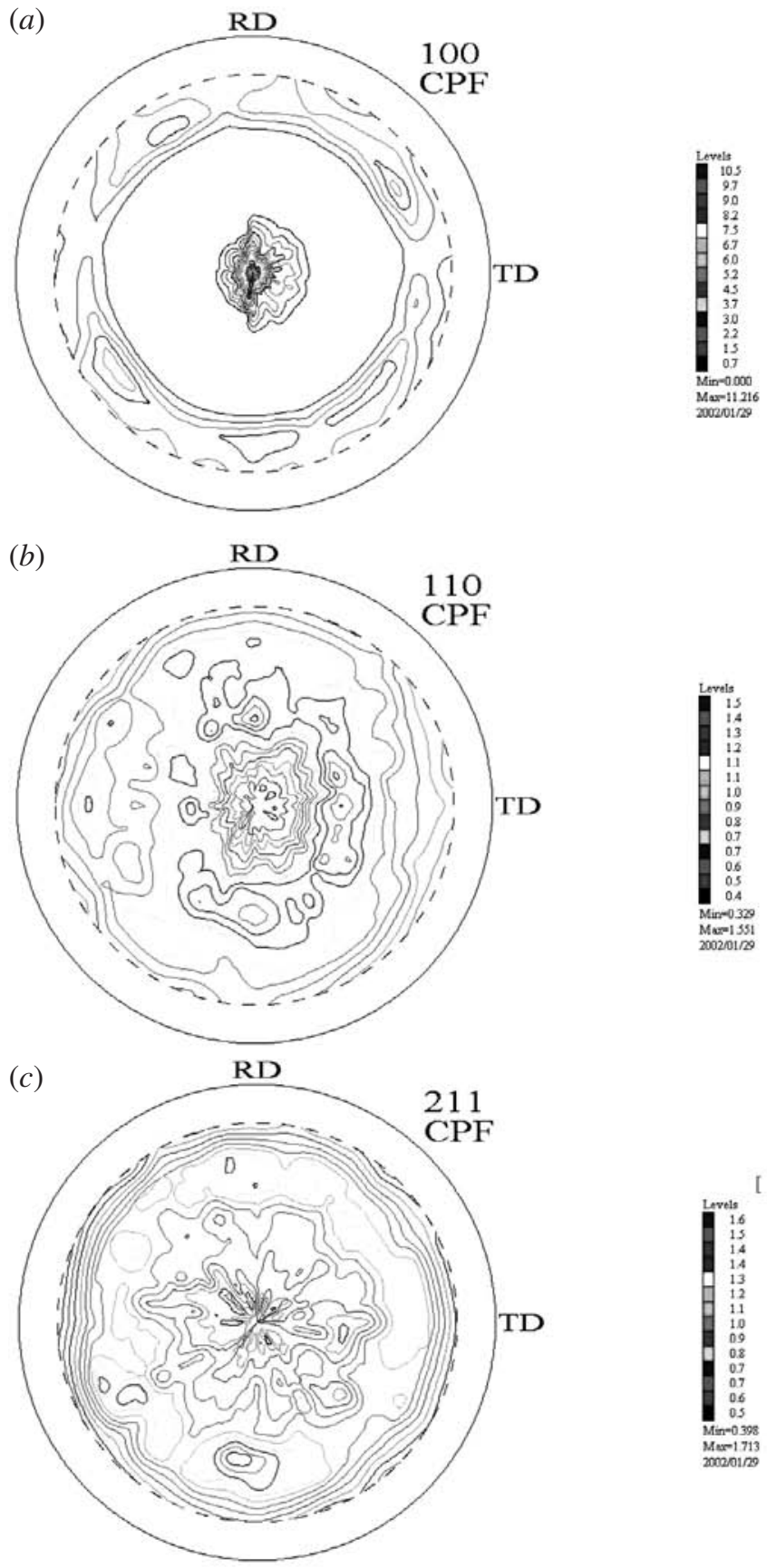

Fig. 8-Pole figures of $(a)\{100\}_{\mathrm{B} 2},(b)\{110\}_{\mathrm{B} 2}$, and $(c)\{211\}_{\mathrm{B} 2}$ registered for the $\mathrm{Cu}-5$ ribbon.

external shape of the ribbons as well as to the wheel rotation direction, revealing a highly textured material. This feature is confirmed by the corresponding pole figures $\{100\}_{\mathrm{B} 2}$ and $\{110\}_{\mathrm{B} 2}$, which are shown in Figure 9. The maximum of normalized intensity of the $\{100\}_{\mathrm{B} 2}$ pole figure concentrates at the center of the figure and reaches a level of 45 , whereas for the $\{110\}_{\mathrm{B} 2}$ pole figure, it is not higher than 1.8. The isolines with weaker intensity at $\{110\}_{\mathrm{B} 2}$ pole figure indicate that some amount of randomly oriented grains is present. These results show that, according to the normalized intensities, the as-received $\mathrm{Cu}-25$ ribbon exhibits a much higher axial texture, mostly $\{100\}_{\mathrm{B} 2}$, than previous ribbons. 


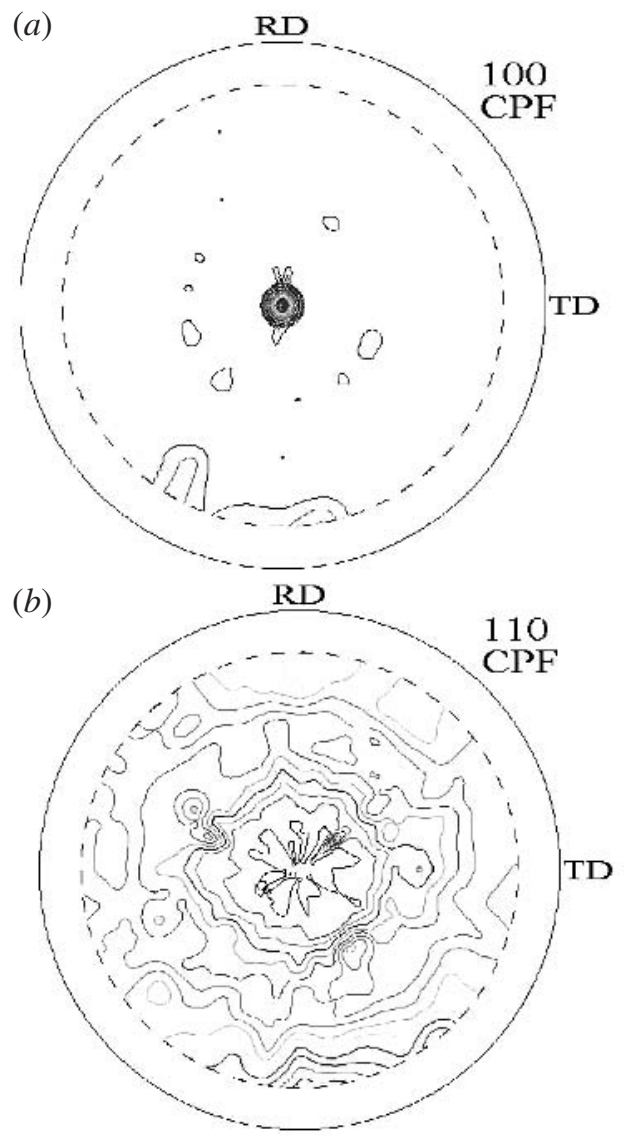

Fig. 9-Pole figures (a) $\{100\}_{\mathrm{B} 2}$ and (b) $\{110\}_{\mathrm{B} 2}$ obtained for the $\mathrm{Cu}-25$ ribbon containing amorphous phase.

As SME properties are to be characterized, a crystallization process has been performed before cycling, by heating the ribbon to $885 \mathrm{~K}$ for 3.5 minutes. The $\mathrm{Cu}-25$ crystallized samples undergo a single-step B2 $\leftrightarrow$ B19 martensitic transformation. After the heat treatment, a mixture of orthorhombic martensite B19 and a small amount of B2 is present at room temperature. The lattice parameters of the orthorhombic martensite were determined from the Rietveld refinement as $a_{0}=2.9252 \AA, b_{0}=4.2842 \AA$, and $c_{0}=4.4772 \AA$, using the orientation described by the Pmmb space group. The $\{100\}_{\mathrm{B} 19}$ and $\{011\}_{\mathrm{B} 19}$ pole figures were registered for the crystallized ribbon as well (Figure 10), where it is clearly visible that the level of normalized intensity rises up to values close to 100 . This particular texture means that grains were growing according to an existing preferred orientation, and therefore $\{011\}_{\mathrm{B} 19}$, axial texture is present. In order to understand this, it is worth noting that the direction of the texture of the crystallized material is the same as that in the as-spun ribbon, according to the crystallographic relationship between the B2 and B19 phases:

$$
[001]_{\mathrm{B} 2} \|[001]_{\mathrm{B} 19}\left[[1 \overline{1} 0]_{\mathrm{B} 2}\left\|[010]_{\mathrm{B} 19} \quad[110]_{\mathrm{B} 2}\right\|[001]_{\mathrm{B} 19}\right.
$$

and so the $\{100\}_{\mathrm{B} 2}$ pole figure refers to the $(011)_{\mathrm{B} 19}$ pole figure in the martensitic phase. The same proceeds in the case of the $\{110\}_{\mathrm{B} 2}$ pole figure, which is converted into (001) for the B19 martensite.

Recent results about the controlled crystallization processes in $\mathrm{Ni}_{25} \mathrm{Ti}_{50} \mathrm{Cu}_{25}$ melt-spun ribbons show that the crystal- (a)
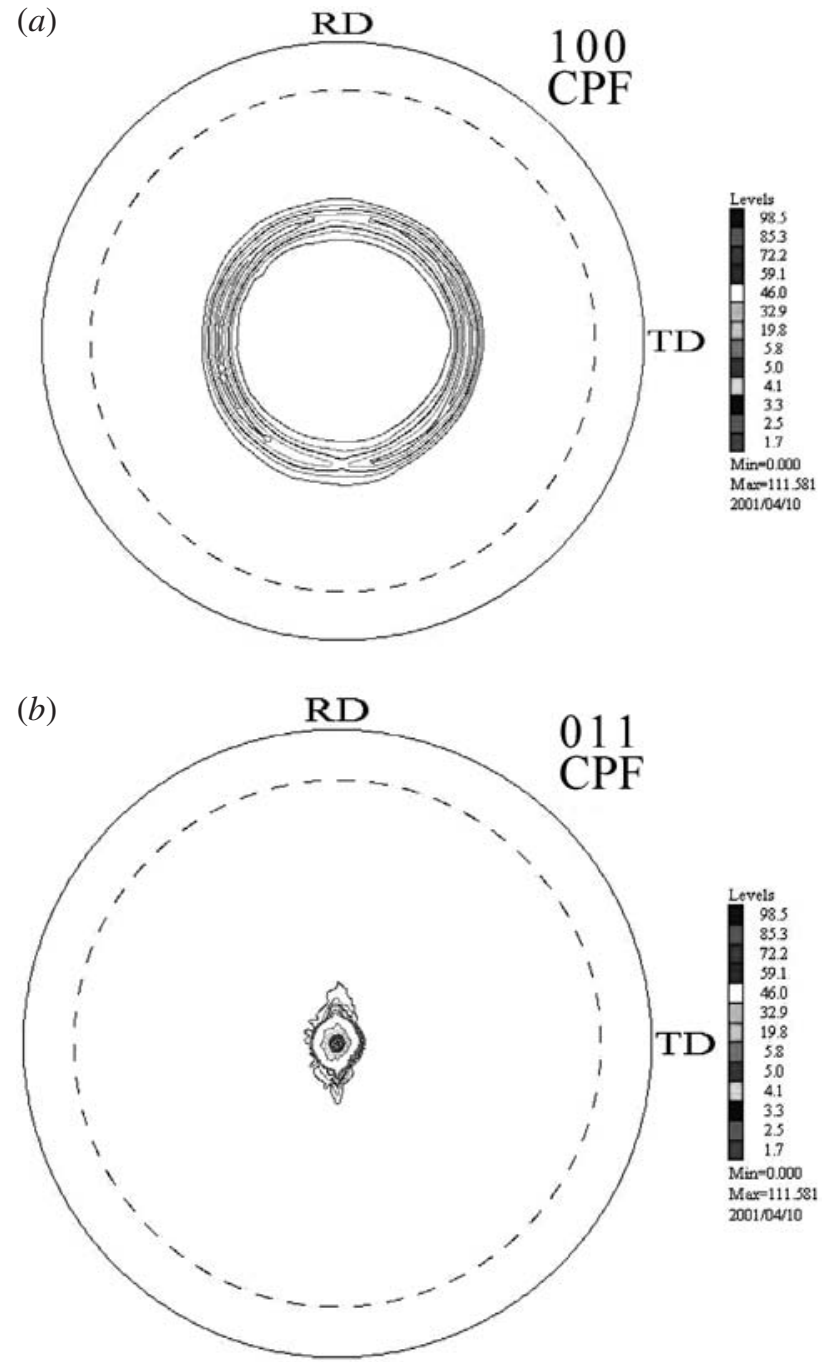

Fig. 10—Pole figures of (a) $\{100\}_{\mathrm{B} 19}$ and (b) $\{011\}_{\mathrm{B} 19}$ registered for the crystallized $\mathrm{Cu}-25$ alloy.

lization produces the grow of the present crystals in such a way that leads to a particular structure consisting of wedgeshaped grains along the thickness of the ribbons, ${ }^{[21]}$ allowing the partial preservation of the initial texture. On the other hand, the observation of a large number of grains with $\{100\}_{\mathrm{B} 2}$ axial texture in all the studied ribbons is possibly related to the preferential orientation of columnar grains that has been observed also in classic melt cast. Casts of bcc metals indeed reveal preferred columnar grains with their central $[100]_{\mathrm{B} 2}$ axis parallel to the direction of the heat flow and thus normal to the mold wall. ${ }^{[22]}$ Translating this into the melt-spinning ribbons, it is reasonable to find a high textured alloy with grains having $\langle 100\rangle_{\mathrm{B} 2}$ perpendicular to the surfaces of the ribbons.

As far as the microstructure is concerned, the as-crystallized $\mathrm{Ni}_{25} \mathrm{Ti}_{50} \mathrm{Cu}_{25}$ samples have different features in relation to the previous alloys, showing rather nonhomogeneous grain sizes (most of the grains are smaller than $1 \mu \mathrm{m}$ and many of them are even below $0.2 \mu \mathrm{m}$ ). These grains are either in austenite or martensite at room temperature, the latter ones usually showing only bivariant or even monovariant structures, probably 


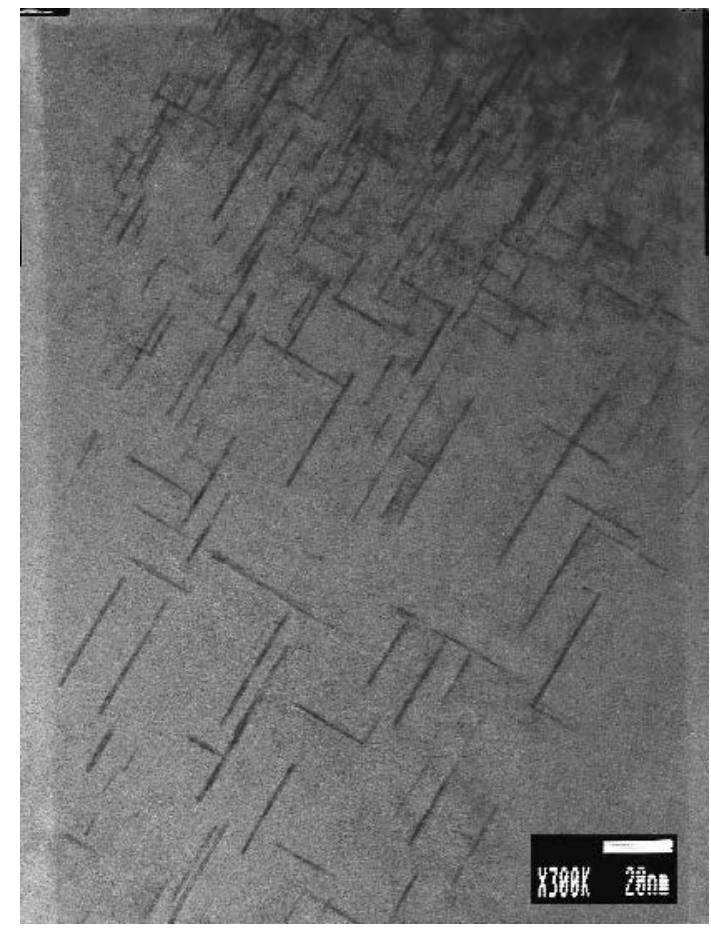

Fig. 11-Bright-field micrograph of the crystallized $\mathrm{Cu}-25$ in austenite showing linear precipitates lying in the $\{100\}_{\mathrm{B} 2}$ planes.

because the local stresses associated to the small grain size make more stable only one preferential orientation in each grain instead of the self-accommodated configuration. Inside the grains, it is possible to observe two kinds of precipitates: a very fine precipitate distribution of a few nanometers in size and linear traces lying on the $\{100\}_{\mathrm{B} 2}$ planes (Figure 11). Deeper studies have been carried out in the latter ones by means of energy-dispersive X-ray microanalysis and electron diffraction, being identified as $\mathrm{TiCu}$ plate like precipitates, with tetragonal structure (B11). Similar precipitates were also found after aging a melt-spun ribbon with the same composition. ${ }^{[23]}$ In addition, the grains appear almost completely free of dislocations.

Thermomechanical cycling shows, as expected, an increase of the recoverable strain as the tensile load increases (Figures 12 and 3), reaching values close to 2.5 pct at $150 \mathrm{MPa}$ (plus a 0.3 pct of permanent strain at this stress). The maximum applied stress, $150 \mathrm{MPa}$, corresponds to the limit given by the brittle fracture stress at room temperature in our experiments. As in the previous alloys, the analysis of the transformation temperatures as a function of the external stress shows a linear dependence. Good agreement between the $d \sigma / d T$ slopes obtained experimentally $(8 \mathrm{MPa} / \mathrm{K})$ and by the Clausius-Clapeyron equation $(8.5$ and $7.9 \mathrm{MPa} / \mathrm{K}$ for Eq. [1] and [2], respectively) exists for the present ribbon. However, it is possible to detect several different features, compared to the previous compositions, concerning the evolution of the thermal cycles under external stress. First of all, the recoverable strain, at similar values of external stress, is notably smaller. In order to explain this effect, the lower deformation of the orthorhombic cell (B19) along a particular direction in comparison to the monoclinic one (B19') has to be considered. This difference is responsible

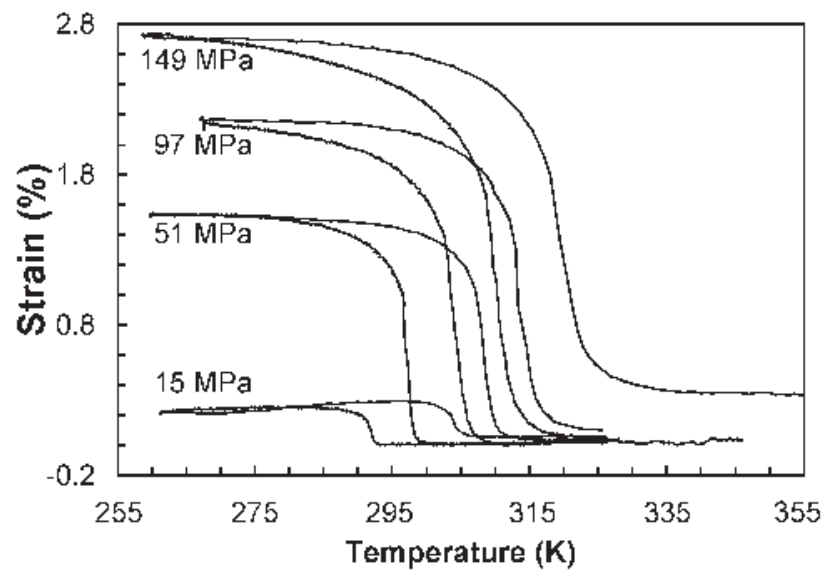

Fig. 12-Selection of thermal cycles performed in the $\mathrm{Cu}-25$ ribbon under increasing stresses.

for the decrease of the macroscopic strain when $\mathrm{Cu}$ content increases, ${ }^{[24,25]}$ as the amount of B19 increases in detriment of B19', then involving lower strains. The maximum recoverable strain obtained experimentally is in good agreement with the values reported in Reference 24 for bulk alloys (with $\mathrm{Cu}$ content from 5 to 20 at. pct) under $90 \mathrm{MPa}$. A small residual deformation appears in bulk samples at higher stresses (close to 0.2 pct at $150 \mathrm{MPa}$ in the 20 at. pct $\mathrm{Cu}$ ). Therefore, the $\mathrm{Cu}-25$ ribbons studied here show similar behavior to bulk alloys, as far as the values of recoverable and permanent deformation under comparable loads are concerned.

Second, a change of the shape in the thermomechanical cycles when increasing external stress has been detected in the single graphs (Figure 12), i.e., they show a long tail at the end of the direct transformation extended toward lower temperatures. This could be related to the likely formation of the B19' phase when applying external stress, also supported by the fact that one small peak in the X-ray spectra of the as-crystallized state cannot be explained either by the B19 or B2 structure, but it matches the most intense peak of B19'. Despite the fact that the manifestation of the B19 or B19' depends on the previous thermal and mechanical processing of the sample, ${ }^{[6]}$ the B19' phase has not been observed frequently, except for the alloys cast by melt spinning, which sometimes present both phases simultaneously. ${ }^{[26]}$ Another possible explanation of the tale of the thermomechanical cycles could be the small size of the grains in the as-crystallized samples as well as the presence of monovariant grains in martensite. The driving force to transform these grains could be higher, needing an extra cooling to transform into the right variant and thus producing the tale of the strain-temperature cycles.

The third considerable difference to take into account is that no TWSME induced by thermomechanical cycling has been detected after 70 cycles with a maximum stress of $120 \mathrm{MPa}$. Despite the fact that the kind of sequence/ transformation for this alloy could play a role in the absence of the TWSME (although in a melt-spun ribbon with the same nominal composition, the effect has been observed indeed ${ }^{[27]}$ ), the particular microstructure of this ribbon could 
inhibit the two-way shape memory due to the presence of the $\mathrm{TiCu}$ phase, ${ }^{[27]}$ to the small size of the grains, or to the absence of a relevant number of dislocations (as will be discussed in Section D).

Before finishing the analysis of the thermomechanical cycling of this ribbon, it is worth taking into consideration the different hysteresis observed in $\mathrm{Cu}-5$ and $\mathrm{Cu}-25$ results. This effect of the $\mathrm{Cu}$ substituting $\mathrm{Ni}$ is already known, as was pointed out previously. However, the reduction observed in $\mathrm{Cu}-25$ ribbons with respect to $\mathrm{Cu}-5$ is not as important as in the corresponding bulk alloys. ${ }^{[25]}$ This effect can be attributed to the smaller grain size in $\mathrm{Cu}-25$ compared to the $\mathrm{Cu}-5$, which grows the interfacial friction involved in completing the transformation and so increases its hysteresis.

Additionally to the thermomechanical cycling, the apparent Young modulus values in martensite (room temperature) and austenite have been estimated by means of tensile tests, yielding 15 and $35 \mathrm{GPa}$, respectively (the reorientation of the variants under external stress being responsible for the smaller apparent elastic modulus in martensite). The corresponding values for bulk material have not been found in the literature for this composition, although for the equiatomic $\mathrm{NiTi}$, they are 20 to $40 \mathrm{GPa}$ in martensite and 40 to $90 \mathrm{GPa}$ in austenite, respectively. ${ }^{[28]}$ These values do not permit further comparisons than to observe the similar magnitude of the modulus and the relative difference between the austenite and martensite.

Tensile tests have been performed to obtain the superelastic effect in the melt-spun ribbons by means of the stress-induced transformation (Figure 13). In this plot, load-unload cycles at increasing strains are shown, up to recoverable strains close to 2.5 pct. A similar value has been obtained by means of thermomechanical cycling, which shows a good functional behavior of the ribbons made by this technique, when the "premature" fracture of fatigue allows it. Brittle fracture is usually obtained at low values of the external stress $(\sim 170 \mathrm{MPa}$ on average for the tensional tests and around $100 \mathrm{MPa}$ for the thermomechanical cycles).

\section{TEM Observations after Thermomechanical Cycling}

All the ribbons have been observed under transmission electron microscopy before and after the thermomechanical

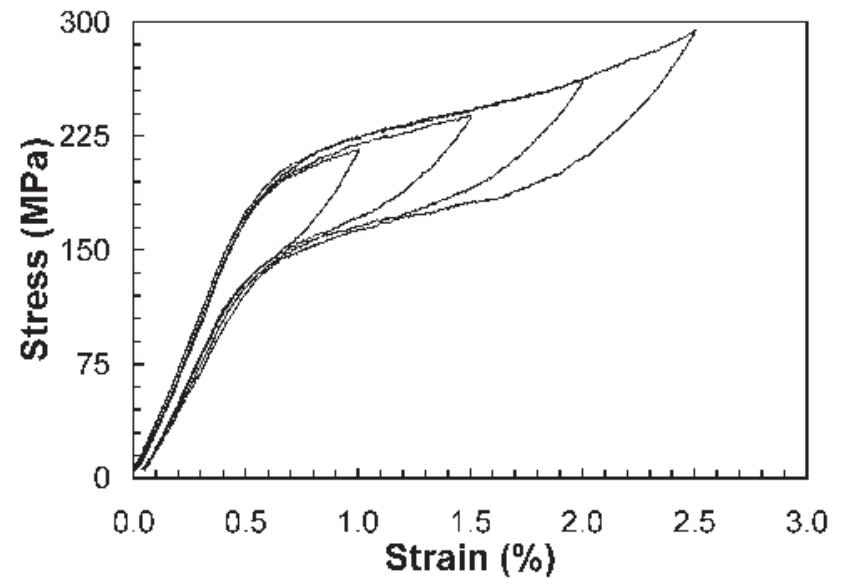

Fig. $13-\sigma-\varepsilon$ plot showing the superelastic behavior of the crystallized $\mathrm{Cu}-25$ ribbon at $320 \mathrm{~K}$. cycling experiments, looking especially at the changes in the configuration of the dislocations. Even in the as-spun state, a high amount of dislocation entanglements together with some small precipitates are present in the NiTi (Figure 14(a)) and $\mathrm{Ni}_{45} \mathrm{Ti}_{50} \mathrm{Cu}_{5}$ (Figure 14(b)) ribbons, as commented on in Sections A and B. After thermomechanical cycling, no big differences have been detected from the TEM observations, i.e., there is neither a clear increase nor decrease in the amount of dislocations (Figures 14(c) and (d)). There can be, at most, a very slight modification in the arrangement of dislocations, although it is difficult to determine accurately due to the high density and complication of the dislocation entanglements existing both before (Figures 14 (a) and (b)) and after (Figures $14(\mathrm{c})$ and $(\mathrm{d}))$ thermomechanical cycling. In any case, the absence of this kind of defect in the as-crystallized $\mathrm{Cu}$ 25 samples (not showing TWSME) indicates the importance of these dislocations for the manifestation of the TWSME in the binary and $\mathrm{Cu}-5$ ribbons.

\section{CONCLUSIONS}

The recoverable strains (SME) obtained in the melt-spun ribbons ( $\sim 4,5$, and 2.5 pct for $\mathrm{NiTi}, \mathrm{Cu}-5$, and $\mathrm{Cu}-25$, respectively) are of the same order as for conventionally cast alloys with similar compositions, studied by other authors. All the ribbons exhibit $\{100\}_{\mathrm{B} 2}$ axial texture. However, the slight tilting of the axial texture in the $\mathrm{Cu}-5$ ribbon can increase the transformation strain in the direction of the applied stress, while the additional presence of sheet texture in the binary ribbon has an opposite effect, which explains the higher strains obtained in the $\mathrm{Cu}-5$ ribbon compared to the binary one and shows the importance of the texture in the amount of SME. On its turn, the lower strain obtained in the $\mathrm{Cu}-25$ ribbon is due to the formation of B19 martensite, which has a lower intrinsic transformation strain. The nonrecoverable strains (lower than 0.3 pct in all cases) show similar values to the ones observed in their corresponding bulk alloys. The TWSME induced after thermal cycling under stress has only been obtained in the NiTi and $\mathrm{Cu}-5$ ribbons.

The entire set of results shows that the alloys made by melt spinning would be a good substitute for the conventionally cast alloys in applications where very flat samples are needed, avoiding the necessity of going through complicated manufacturing processes. However, the fracture stresses are significantly lower than in corresponding bulk alloys, due to the irregularities in the thickness of the obtained ribbons. This points to the need for an enhancement of the quality of the ribbons to be used under higher axial stresses.

\section{ACKNOWLEDGMENTS}

Financial support to R. Santamarta by means of a grant from the Universitat de les Illes Balears (UIB) is acknowledged. Partial financial support from DGI (Project Nos. MAT2000-0862-C03-02 and MAT2002-00319) is also acknowledged. The authors thank Professor R. Portier, Dr. P. Ochin, Dr. V. Kolomytsev, Mr. A. Dezellus, and Mr. P. Plaindoux for the fabrication of the alloys used in this study. 

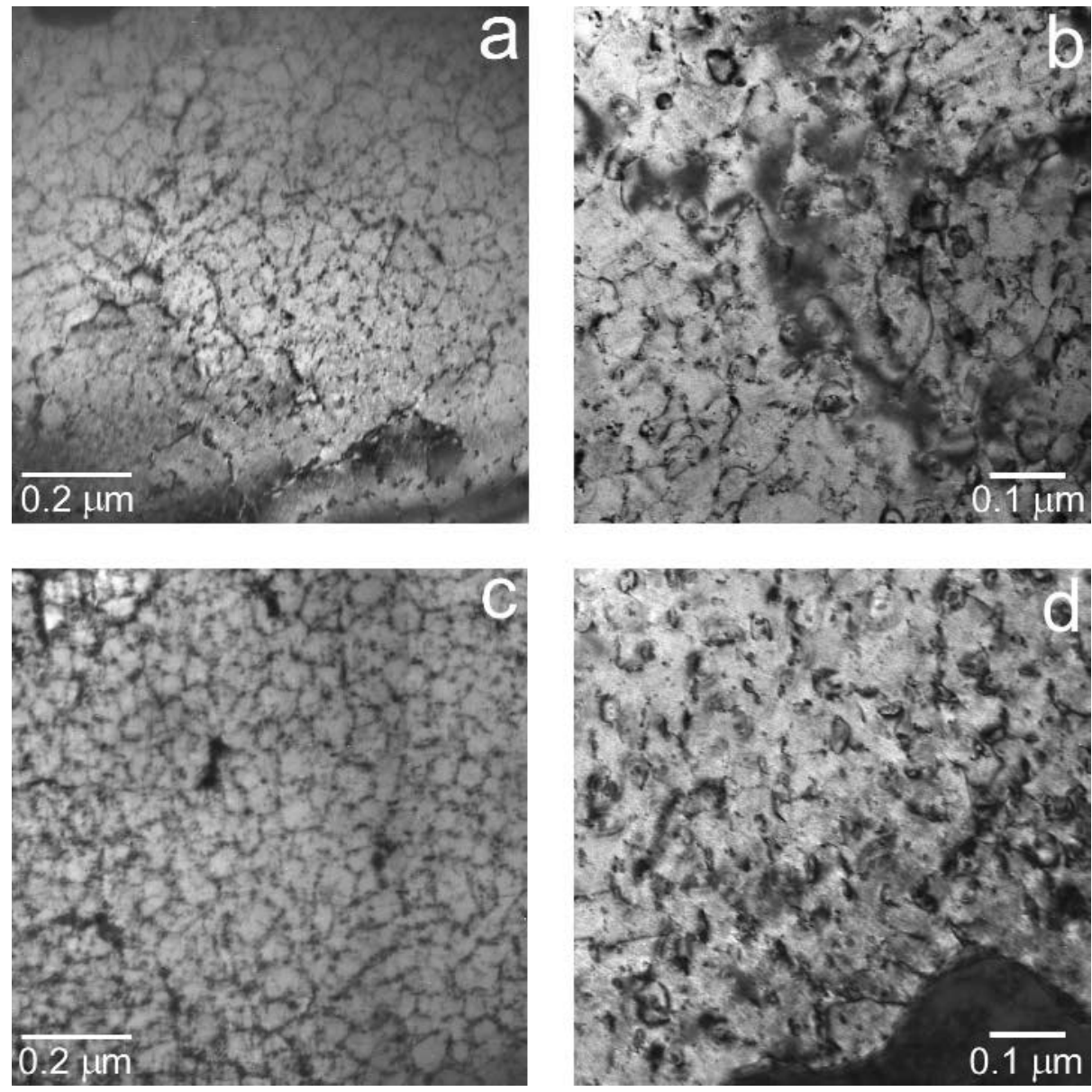

Fig. 14-Bright-field images from the as-spun $(a) \mathrm{NiTi}$ and $(b) \mathrm{Cu}-5$ melt-spun ribbons. $(c)$ NiTi after 140 cycles up to $60 \mathrm{MPa}$ and $(d) \mathrm{Cu}-5$ after 105 cycles up to $80 \mathrm{MPa}$.

\section{REFERENCES}

1. J. Beyer and J.H. Mulder: Mater. Res. Soc. Symp. Proc., 1995, vol. 360, pp. 443-54.

2. H. Hosoda, T. Fukul, K. Inoue, Y. Mishima, and T. Suzuki: Mater. Res. Soc. Symp. Proc., 1997, vol. 459, pp. 287-93.

3. V.A. Lobodyuk and M.M. Medyukh: Met. Phys. Adv. Technol., 1997 vol. 16, pp. 493-503

4. M. Piao, S. Miyazaki, K. Otsuka, and N. Nishida: Mater. Trans. JIM, 1992, vol. 33, pp. 337-45.

5. K. Otsuka and C.M. Wayman: Shape Memory Materials, 1st ed., Cambridge University Press, Cambridge, United Kingdom, 1998, pp. 49-96.

6. W.J. Moberly and K.N. Melton: Engineering Aspects of Shape Memory Alloys, 1st ed., Butterworth-Heinemann Ltd., Essex, 1990, pp. 46-57.

7. D.R. Angst, P.E. Thoma, and M.Y. Kao: J. Phys. IV, 1995, vol. C8, pp. $747-52$

8. S.F. Hsieh and S.K. Wu: Mater. Characterization, 1998, vol. 41 pp. 151-62.

9. R. Santamarta, C. Seguí, J. Pons, and E. Cesari: Scripta Mater., 1999 , vol. 41 , pp. $867-72$

10. E. Cesari, J. Pons, R. Santamarta, C. Seguí, D. Stróz, and H. Morawiec: Proc. XVIII Conf. on Applied Crystallography, World Scientific, Singapore, 2001, pp. 171-85.

11. Y. Furuya, M. Matsumoto, and T. Matsumoto: Proc. Int. Conf. on Martensitic Transformation, Monterey Institute for Advanced Studies, Monterey, CA, 1993, pp. 905-09.

12. S.D. Wang, X.Z. Wu, J.P. Zhang, H.Q. Su, J.L. Jin, and B.T. Song: Trans. Mater. Res. Soc. Jpn., 1994, vol. 18B, pp. 1061-64.
13. R. Santamarta, E. Cesari, J. Pons, C. Seguí, P. Ochin, and R. Portier: Proc. Solid-Solid Phase Transformation '99, The Japan Institute of Metals, Sendai, 1999, pp. 1076-79.

14. P. Wollants, M. De Bonte, and J.R. Roos: Z. Metallkd., 1979, vol. 70, pp. 113-17.

15. S. Miyazaki and K. Otsuka: Metall. Trans. A, 1986, vol. 17A, pp. 53-63.

16. C.B. Stachowiak and P.G. McCormick: Acta Metall., 1988, vol. 36, pp. 291-97.

17. P. Sittner and V. Novák: Int. J. Plasticity, 2000, vol. 16, pp. 1243-68.

18. K. Bhattacharya and R.V. Kohn: Acta Mater., 1996, vol. 44 pp. $529-42$.

19. P. Olier, J.C. Brachet, J.L. Bechade, C. Foucher, and G. Guénin: J. Phys. IV, 1995, vol. C8, pp. 741-46.

20. G. Airoldi, T. Ranucci, G. Riva, and A. Sciacca: J. Phys.-Condens. Mat., 1995, vol. 7, pp. 3709-20.

21. T. Goryczka: Ph.D. Thesis, University of Silesia, Katowice, 2001.

22. C.S. Barret and T.B. Massalski: Structure of Metals, 3rd ed., McGrawHill, New York, NY, 1966.

23. H. Rösner, P. Schlosmacher, A.V. Sheliakov, and A.M. Glezer: Scripta Mater., 2000, vol. 43, pp. 871-76.

24. T.H. Nam, T. Saburi, and K. Shimizu: Mater. Trans. JIM, 1990, vol. 31, pp. 959-67.

25. T.H. Nam, T. Saburi, Y. Nakata, and K. Shimizu: Mater. Trans. JIM, 1990, vol. 31, pp. 1050-56.

26. T. Goryczka, M. Karolus, P. Ochin, and H. Morawiec: J. Phys. IV, 2001, vol. 11, pp. 345-50

27. A.V. Shelyakov, Y.A. Bykovsky, N.M. Matveeva, and Y.K. Kovneristy: J. Phys. IV, 1995, vol. C8, pp. 713-16.

28. Y. Liu and H. Yang: Mater. Sci. Eng. A, 1999, vol. A260, pp. 240-45. 